\title{
Pyoderma Gangrenosum with Positive Antinuclear Antibody, in the Absence of Systemic Association
}

\author{
Shrestha $\mathbf{S}^{1}$, Aryal $\mathbf{A}^{2}$ \\ ${ }^{1}$ Lecturer, ${ }^{2}$ Second Year Resident, Department of Dermatology, Dhulikhel Hospital, Kathmandu University-Teaching \\ Hospital, Dhulikhel, Kavre, Nepal.
}

\begin{abstract}
Pyoderma gangrenosum is an uncommon neutrophilic dermatosis, seen on legs, and infrequently on hands and other anatomical sites. It is associated with systemic diseases in $50-70 \%$ of the cases. Antinuclear antibody (ANA) seropositivity has been reported in pyoderma gangrenosum associated with connective tissue disorders. However, there are very few case reports of pyoderma gangrenosum in patients of systemic lupus erythematosus, while we did not find any reports of ANA seropositivity in isolated pyoderma gangrenosum. Hence, we report this unique case of pyoderma gangrenosum with classical clinicohistopathology, positive ANA but no systemic association. As anticipated, our patient responded promptly to steroids.
\end{abstract}

Key words: Antibodies; connective tissue diseases; lupus erythematosus; vasculitis, leukocytoclastic

\section{Introduction}

$\mathrm{P}$ yoderma gangrenosum (PG) is a rare necrotizing, ulcerative neutrophilic dermatosis. ${ }^{1}$ It is usually associated with various systemic illnesses, but rarely described in association with systemic lupus erythematosus (SLE) or antinuclear antibody (ANA) seropositivity. We report this case of PG on sunexposed sites, with positive ANA and no internal disease.

\section{Case Report}

A 61 years old lady, presented to Dermatology OPD, with complaint of multiple painful ulcers on dorsa of bilateral hands and feet for seven days. Initially, there were multiple, asymptomatic, erythematous papules on hands and feet, that rapidly evolved into large ulcerative plaques. Patient did not notice any constitutional symptoms during progression of lesions. She had no prior history of trauma, insect bite, topical application or drug intake. There was no fever, joint pain, morning stiffness, diarrhea, constipation, pain abdomen, blood in stool, weight loss or decreased appetite. Patient did not have oral ulcers, malar rash, hair loss, menstrual irregularity or headache. No

Address of Correspondence:

Dr. Smriti Shrestha

Dhulikhel Hospital, Kathmandu University-Teaching Hospital,

Dhulikhel, Kavre, Nepal.

E-mail: smiaaliyah@gmail.com systemic comorbidities were elicited from history. She was a nonsmoker.

On examination, patient was afebrile with normal vital signs (BP 100/60 mm of mercury, Pulse 84/m, RR $18 / \mathrm{min})$. Cutaneous examination revealed five annular lesions on sun-exposed sites of hands and feet (Figure 1). Among them, only one lesion on right hand was vegetative. Others were ulcerative plaques of varying sizes, with undermined edges, irregular border, containing hemorrhagic and necrotic slough and a conspicuous edematous violaceous margin. Largest plaque had dimensions of $6 \times 5 \mathrm{~cm}$. Head to toe examination and systemic findings were normal. Pathergy test was negative.

Submitted: $5^{\text {th }}$ January 2018

Accepted: $18^{\text {th }}$ February 2018

Published: $21^{\text {st }}$ March 2018

How to cite this article

Shrestha S, Aryal A. Pyoderma gangrenosum with positive antinuclear antibody, in the absence of systemic association. Nepal Journal of Dermatology Venereology and Leprology. 2018;16(1):66-9. doi:http://dx.doi.org/10.3126/njdvl.v16i1.19418

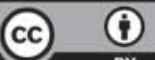

Licensed under CC BY 4.0 International License which permits use, distribution and reproduction in any medium, provided the original work is properly cited. 
Blood tests and routine investigations were sent. There was mild leukocytosis of $10,300 \times 10^{9} / \mathrm{L}$ (reference interval $\left.4.0 \times 0^{9}-11.0 \times 10^{9} / \mathrm{L}\right)$ with neutrophilia (82\%), and elevated acute phase reactants, ESR $44 \mathrm{~mm} / \mathrm{hr}$ (reference interval 0-20 mm/hour) and CRP 31 $\mathrm{gg} /$ $\mathrm{ml}$ (reference interval $0-5 \mu \mathrm{g} / \mathrm{ml}$ ). Peripheral smear showed shift to left with neutrophilia, toxic granules and RBC polychromatophils. Antinuclear antibody was raised as 3.1 (reference interval 0.5-1.0 IU) However, investigations done for blood sugar, liver function, renal parameters, urine and stool routine, stool for occult blood, rheumatoid factor, anti-ds DNA, serum complement, thyroid profile, wound swab culture, abdominal ultrasonography and colonoscopy were normal.

Histopathology confirmed our provisional diagnosis, showing epidermal intracorneal neutrophilic collections with areas of necrosis and extensive dermal neutrophilic infiltrates with vasculopathy (Figure 2).

Oral steroids (prednisolone) was started upon admission. Normal saline and vaseline gauze dressing was done twice a day for 3 days. Skin lesions showed dramatic improvement with steroids within 48 hours (Figure 3). Patient was kept on hydroxychloroqine as maintenance therapy. There was no relapse until two months of discontinuation of therapy (till date).
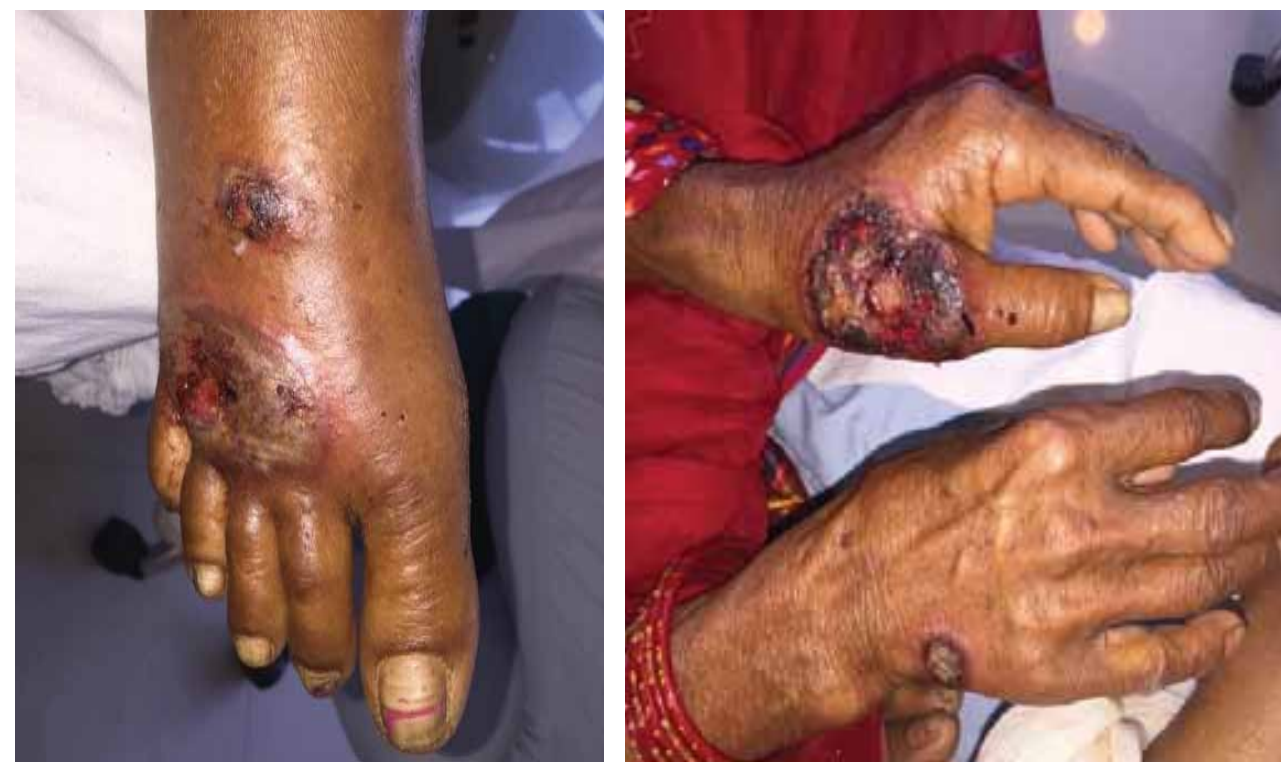

Figure 1: Large ulceronecrotic lesions with edematous violaceous border on dorsa of hands and feet. Single vegetative lesion on dorsum of right hand
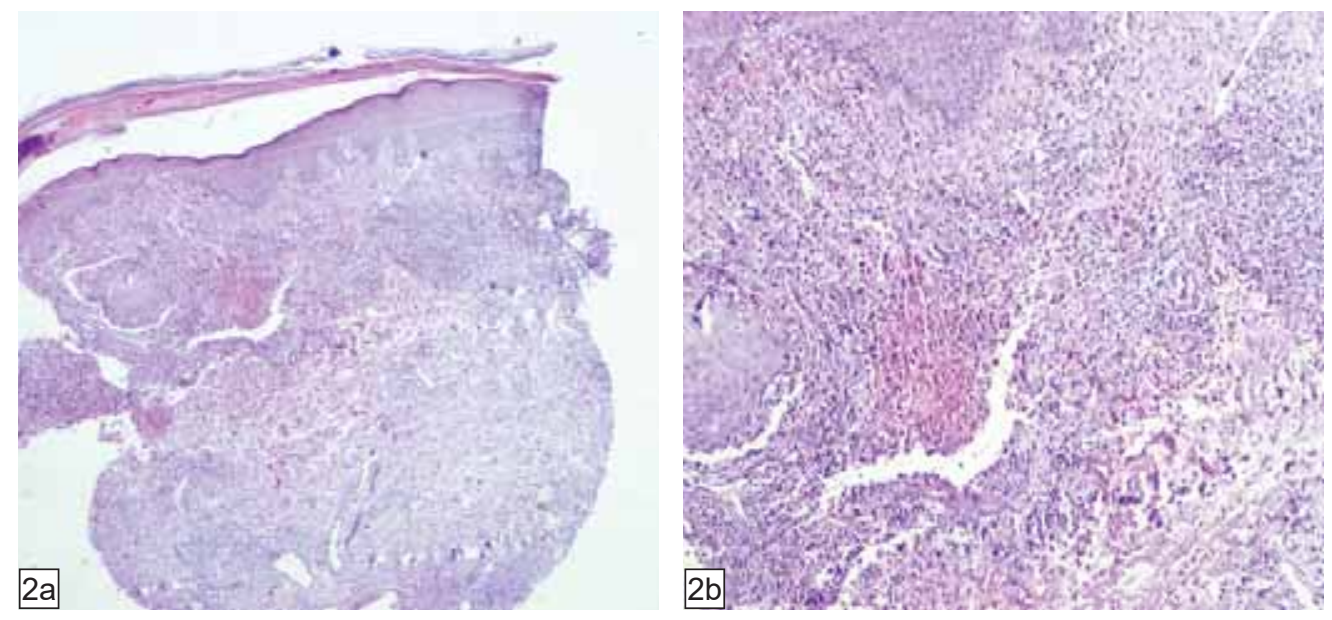

Figure 2a, 2b: Hematoxylin and Eosin stain: 2a (4x), 2b (10x) photomicrograph showing epidermal hyperkeratosis, irregular acanthosis and spongiosis with foci of intracorneal neutrophilic collections and areas of necrosis. Papillary dermis shows extensive neutrophilic infiltration forming abscess, with fragmented neutrophils, suppurative necrosis, hemorrhage and endothelial swelling. Lower dermis has mixed infiltrates of perivascular and periadnexal neutrophils and lymphocytes. No evidence of leukocytoclastic vasculitis. 

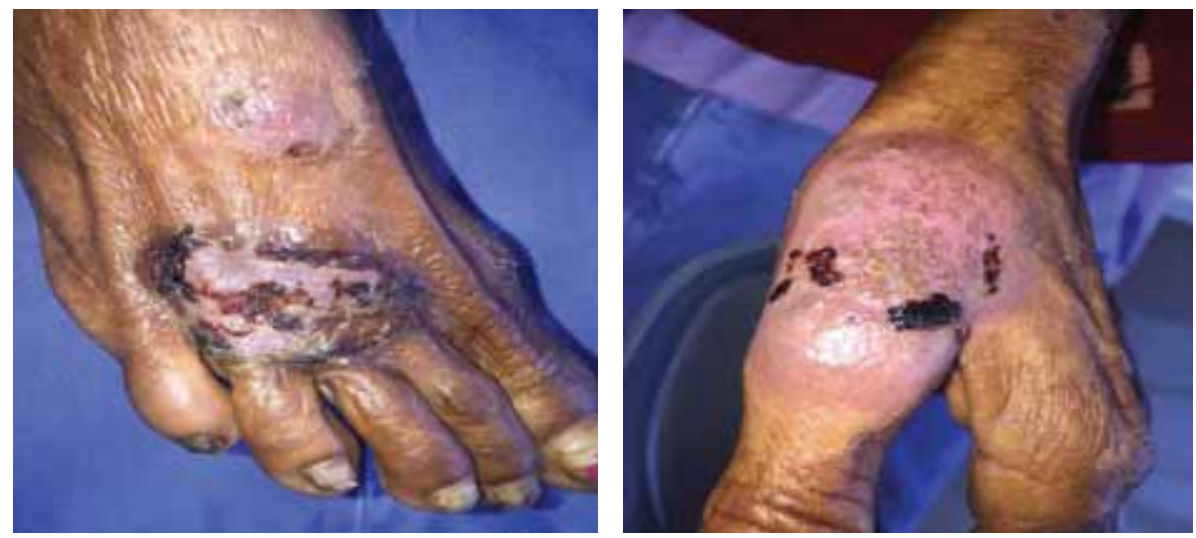

Figure 3: Clinical improvement on Day 2 and Day 4 of starting treatment.

\section{Disaussion}

Pyoderma gangrenosum (PG) is a painful, noninfectious, rapidly spreading, necrotizing skin ulceration, regarded as a spectrum of neutrophilic dermatosis. ${ }^{1}$ It was first described in 1908 by French dermatologist Brocq as "geometric phagedenism", and later Brunsting et al renamed it as "pyoderma gangrenosum" as he believed that it occurred secondary to bacterial infection. ${ }^{2,3}$

It is an uncommon reactive phenomenon, with 3-10 new cases per million population yearly. ${ }^{4}$ It usually affects adults between $20-50$ years and rarely children. Around $50-70 \%$ cases have systemic association, observed commonly with inflammatory bowel disease, rheumatoid arthritis and hematologic malignancy. It is also seen in surgical wound sites and at sites of trauma, known as pathergy, which occurs in $25-30 \%$ of PG. ${ }^{1,4}$

There are four clinical forms of PG described as ulcerative, bullous, pustular and vegetative lesions. Various clinical subtypes have distinct systemic association. It begins as inflammatory papule, nodule or pustule, which swiftly develops into undermined ulcero-necrosis with characteristic violaceous border. ${ }^{1}$ It heals with descriptive cribiform "cigarette paperlike" scars. ${ }^{5}$ Lesions occur in leg in $70 \%$ cases, less common on face, neck, hands, trunks, breast, genitalia and rarely at extracutaneous tissues. ${ }^{6,7}$ We did not find pyoderma gangrenosum reported on sun-exposed sites.

Histopathology of early lesion from advancing border shows inflammatory infiltrates in dermis, with or without leukocytoclastic vasculitis. Ulceration of the epidermis tends to be secondary to the dermal inflammation. Older lesion with ulceration shows neutrophilic infiltrates in epidermis and dermis, without infectious agents. ${ }^{8}$ Diffuse dermal neutrophilic infiltrates seen in PG, without vasculitis and granuloma, has been called "sea of neutrophils." ${ }^{1}$

In 2004, Su WP et al proposed a diagnostic criterion of PG. ${ }^{5}$ It encompassed characteristic clinical ulcer and histological findings, in association with systemic illnesses, after excluding other causes of cutaneous ulceration. Resistance to antibiotics and surgical therapy and improvement with steroids is distinctive. ${ }^{7}$ In our patient, there were classical lesions of PG on sun-exposed sites, that responded effectively to steroids. Diagnosis of PG was made after exclusion of other causes of cutaneous ulcer. Seropositivity for ANA without manifestations of SLE or any other systemic disease is unprecedented.

ANA is regarded as a marker of systemic autoimmune connective tissue disorder. Although it is highly (95\%) sensitive for SLE, it is not a specific marker. Positive predictive value of ANA in SLE in 11-49\% and much lower in other connective tissue disorders (CTD)..$^{9}$ On the other hand, ANA is also observed in $5 \%$ of healthy individuals. ${ }^{10}$ Likewise, although SLE is common among women of reproductive age group, pyoderma gangrenosum is a rare entity. Association of PG and SLE is relatively recent and sparse. ${ }^{11}$ Waldman MA et al reported an interesting case where PG preceded onset of SLE by 8 years. ${ }^{12}$ This should be noted as PG might be a harbinger of chronic autoimmunity. At the other end of the fringe, patient with chronic SLE has developed PG lesions. ${ }^{13}$ Hence the temporal relationship between two diseases has been variable. In our case scenario, presence of PG lesions in a female with ANA reactivity suggests possibility of connective tissue disorder in future. However, since ANA is also observed in healthy individuals, and this finding could be coincidental in isolated PG. With further studies, the association of SLE and ANA with PG might be extrapolated in future.

The mainstay of treatment is steroids, although there 
are no standard guidelines yet. Steroid sparing immune modifiers used in PG are azathioprine, cyclosporine, cyclophosphamide, mycophenolate mofetil, biologics and intravenous immunotherapy. ${ }^{1,3,6}$

\section{Condusion}

Given the rarity of pyoderma gangrenosum, and no previous reports of ANA reactivity without systemic association, we have described this entity. We did not find any evidence of connective tissue disorder in our patient, but have discussed previous associations. Further studies might validate the relation of PG with SLE, as well as its dynamics with ANA serology.

Financial disclosure: None.

Conflicts of interest to disclosure: None declared.

\section{References}

1. Bhat RM. Pyoderma gangrenosum: An update. Indian Dermatol Online J. 2012;3(1):7-13. https://doi.org/10.4103/2229-5178.93482

2. Brocq L. A new contribution to the study of geometric phagedenism. Ann Dermatol Syphiligr. 1916;9:1-39.

3. Bhat RM, Nandakishore B, Sequeira FF, Sukumar D, Kamath GH, Martis J, et al. Pyoderma gangrenosum: An Indian perspective. Clin Exp Dermatol. 2011;36:242-7. https://doi. org/10.1111/j.1365-2230.2010.03941.x

4. Wollina U. Pyoderma gangrenosum - a review. Orphanet J Rare Dis. 2007;2:19. https://doi. org/10.1186/1750-1172-2-19

5. Su WP, Davis MD, Weenig RH, Powell FC, Perry HO. Int J Dermatol. 2004;43(11):790-800. https:// doi.org/10.1111/j.1365-4632.2004.02128.x

6. Satoh M, Yamamoto T. Genital pyoderma gangrenosum: report of two cases and published work review of Japanese cases. J Dermatol. 2013;40(10):840-3. https://doi. org/10.1111/1346-8138.12252

7. Marinopoulos S, Theofanakis C, Zacharouli T, Sotiropoulou M, Dimitrakakis C. Pyoderma Gangrenosum of the breast: A case report study. Int J Surg Case Rep. 2017;31:203-5. https://doi. org/10.1016/j.ijscr.2017.01.036

8. Su WP, Schroeter AL, Perry HO, Powell FC. Histopathologic and immunopathologic study of pyoderma gangrenosum. J Cutan Pathol. 1986;13(5):323-30. https://doi. org/10.1111/j.1600-0560.1986.tb00466.x

9. Soto $M E$, Hernandez-Becerril $N$, Perez-Chiney AC, Hernandez-Rizo A, Telich-Tarriba JE, JuarezOrozco Le, et al. Predictive value of antinuclear antibodies in autoimmune diseases classified by clinical criteria: Analytical study in a specialized health institute, one year followup. Results Immunol. 2015;5:13-22. https://doi. org/10.1016/j.rinim.2013.10.003

10. Satoh M, Mercado MV-D, Chan EKL. Clinical interpretation of antinuclear antibody tests in systemic rheumatic diseases. J Modern Rheumatol. 2009;19(3):219-28. https://doi. org/10.3109/s10165-009-0155-3

11. González-Moreno J, Ruíz-Ruigomez M, Callejas Rubio JL, Ríos Fernández R, Ortego Centeno N. Pyoderma gangrenosum and systemic lupus erythematosus: a report of five cases and review of the literature. Lupus. 2015;24(2):130-7. https://doi.org/10.1177/0961203314550227

12. Waldman MA, Callen JP. Pyoderma gangrenosum preceding the diagnosis of systemic lupus erythematosus. Dermatol. 2005;210(1):64-7. https://doi.org/10.1159/000081488

13. Pinto GM, Cabecas MA, Riscado M, Goncalves $\mathrm{H}$. Pyoderma gangrenosum associated with systemic lupus erythematosus: response to pulse steroid therapy. J Am Acad Dermatol. 1991:24(5):818-21. https://doi.org/10.1016/0190-9622(91)70122-I 